\title{
Glucosamine Enhances Tissue Regeneration in the Process of Wound Healing in Rats as Animal Model: A Stereological Study
}

Soheil Ashkani-Esfahani ${ }^{1}$, Yasaman Emami ${ }^{1}$, Elmira Esmaeilzadeh ${ }^{1}$, Fereshteh Bagheri* and Mohammad Reza Namazi ${ }^{2}$

${ }^{1}$ Student Research Committee, Shiraz University of Medical Sciences, Shiraz, Iran

${ }^{2}$ Department of Dermatology, Shiraz University of Medical Sciences, Shiraz, Iran

\begin{abstract}
Introduction: Glucosamine (GA), a water soluble hexosamine made from chitin or chitosan, was showed to have stimulatory effect on matrix formation, enhancing inflammatory response, modulating hyaluronic acid synthesis which promotes extracellular matrix remodeling leading to better wound healing. GA was also shown to have antioxidant and immunomodulatory effect which play roles in wound healing process. In this study we aimed to determine the effect of topical administration of GA on wound healing process in rats as animal model.

Material and methods: 36 Wistar rats were divided into 3 groups; the control group which received no treatment; the Glucosamine group that received GA gel with $2 \%$ GA concentration, and the Base group that was treated with the vehicle. A $1 \mathrm{~cm}$ full-thickness wound was created on the posterior of each rat's neck. Treatments took place every 24 hours for 15 days. The wound closure rate, volume density of collagen bundles and vessels, fibroblast population, length density and mean diameter of the vessels were estimated by using unbiased stereological and histomorphometrical methods.

Results: GA enhanced the wound closure rate consequently as well as fibroblast proliferation, collagen synthesis and proliferation of hair follicles in contrast with the base and the control group. Although not statistically significant, GA also improved the revascularization process in the wound site.

Conclusion: Results of this study indicate that GA has the potential for being used for treatment of skin wounds; however, still further evaluations on its mechanisms of action and clinical advantages and disadvantages should be performed.
\end{abstract}

Keywords: Glucosamine; Wound healing; Stereology; Rat

\section{Introduction}

The wound healing process is consisted of several components including fibroblast activation and migration, reepithelization, endothelial cell proliferation, and angiogenesis, all along with inflammatory response and oxidative reaction in the damaged site [1]. The wound repair and the tissue regeneration process begins immediately after the injury by the release of different growth factors, cytokines, and etc [2]. In addition, some complications such as disruption of blood vessels, infection and advanced inflammation might occur during the repair process $[3,4]$. Conclusively, intervening in any step of the healing process may affect the rate and quality of tissue regeneration.

Glucosamine (GA) is a hexosamine product made from chitin (which is extensively distributed in nature and is the second most plentiful polymer after cellulose) or chitosan (a cationic polysaccharide derived from $\mathrm{N}$-deacetylation of chitin by hydrolysis) and is a watersoluble material [5]. Many biological, nutrimental and pharmaceutical effects of GA, such as membrane stabilization, liver protection [6], wound healing promotion by enhancing the synthesis of hyaluronic acid [7], and application in osteoporosis treatment [8] have been previously reported. Recently GA is receiving more attention because of its facile absorption and medicinal properties. GA anti-inflammatory activity has been extensively investigated on active inflammatory states such as osteoarthritis [9]. GA also exhibited multiple antioxidant effect as it showed noticeable reducing power and the ability of superoxide/ hydroxyl-radical scavenging in vitro [10] and in vivo as well as immune modulatory impact [11]. Several studies, which mostly focused on oral and systemic administration of GA, investigated its beneficial effects on skin damages, [12] while investigations on wound healing effects of topical GA administration are still lacking. However, according to previous studies, GA was demonstrated to accelerate the wound healing and tissue regeneration rate as well as increasing the moisture content, and reducing wrinkle and scar formation [7,13-15].

In this study, we aimed to determine the effects of topical administration of $2 \%$ GA-gel on full thickness skin wounds in laboratory rats by using stereological methods for evaluation of the wound closure rate as well as quantitative estimation of vascularization, collagen bundle synthesis, hair follicle production, and fibroblast proliferation.

\section{Materials and Methods}

\section{Preparation of creams}

D-(+)-Glucosamine (GA) was supplied by Sigma ${ }^{\mathrm{Tw}}$ (St Quentin Fallavier, France). In order to facilitate the application of the agent, we provided $2 \%, 4 \%$ and $8 \%$ GA gel by dissolving $2 \mathrm{~g}, 4 \mathrm{~g}$, and $8 \mathrm{~g} \mathrm{GA}$, respectively in $2 \mathrm{cc}$ distilled water and then transferred the solution into $2 \%$ carboxymethylcellulose (CMC) $(2 \mathrm{~g} \mathrm{CMC}$ dissolved in $98 \mathrm{cc}$

*Corresponding author: Fereshteh Bagheri, Student Research Committee, Shiraz Medical School, Shiraz University of Medical Sciences, Shiraz, Iran, Tel: +98-9176046722; E-mail: bagheri.fereshte@yahoo.com

Received June 14, 2012; Accepted July 16, 2012; Published July 18, 2012

Citation: Ashkani-Esfahani S, Emami Y, Esmaeilzadeh E, Bagheri F, Namazi MR (2012) Glucosamine Enhances Tissue Regeneration in the Process of Wound Healing in Rats as Animal Model: A Stereological Study. J Cytol Histol 3:150. doi:10.4172/2157-7099.1000150

Copyright: (c) 2012 Ashkani-Esfahani S, et al. This is an open-access article distributed under the terms of the Creative Commons Attribution License, which permits unrestricted use, distribution, and reproduction in any medium, provided the original author and source are credited. 
Citation: Ashkani-Esfahani S, Emami Y, Esmaeilzadeh E, Bagheri F, Namazi MR (2012) Glucosamine Enhances Tissue Regeneration in the Process of Wound Healing in Rats as Animal Model: A Stereological Study. J Cytol Histol 3:150. doi:10.4172/2157-7099.1000150

distilled water). The gel base was also supplied by creating $2 \%$ CMC gel without the GA component.

The pilot study: To determine the dose-dependent effect of GA on wound closure rate, a pilot study was conducted with three groups, numbered from 1 to 3 , each consisted of eight rats with a $1 \mathrm{~cm}^{2}$ wound on the posterior surface of their neck $(n=8)$. The $2 \%, 4 \%$, and $8 \%$ GA gels was topically administered to groups 1 to 3 , respectively, and the mean wound closure rate in each group was determined during a 12 days time period. The wound closure rates in the groups 1,2 , and 3 were $6.45 \% /$ day, $7.21 \% /$ day, and $7.67 \% /$ day, respectively. Since there was statistically insignificant difference between the three groups $(\mathrm{P}=0.9)$, the lowest dosage, $2 \% \mathrm{GA}$ gel, was selected for conducting the main study.

Animals and excision of wound model: In a randomized experimental study, 36 male Sprague-Dawley rats $(200 \pm 20 \mathrm{~g})$ between 2 to 3 months age were randomly divided into three groups of 12 . One group was treated with vehicle gel (Gel base). The second group was treated with 2\% GA gel (Glucosamine), and the third wounded group labeled as the control group which received no treatment. On the day 0 , under general anesthesia, a $1 \mathrm{~cm}^{2}$ circular full-thickness wound was created on the posterior surface of each rat's neck. The debridement procedure was done in a standard way for all of the animals just after the wounding and repeated every 24 hours until the end of the study (the day in which at least one wound in any group of rats was completely closed).

After 15 days, the animals were sacrificed with a high dose of ether and full thickness skin biopsies $(1 \mathrm{~cm} \times 1 \mathrm{~cm})$ were taken from the wound site and fixed in buffered formaldehyde $(\mathrm{pH}=7.2)$ for histomorphometrical and stereological evaluations.

The study protocol was approved by the Animal Ethics Committee of Shiraz University of Medical Sciences and the animal care was in accordance with their guidelines.

Stereological study: To determine the rate of the wound closure, digital photographs were captured from the wound surfaces every four days with a single lens digital camera. To calibrate the magnification of photographs, a standard ruler was set at the level of the wound in each photograph, and the wound area $\left(\mathrm{mm}^{2}\right)$ at each visit was estimated by using a software composed of a point grid, designed at our research centre (Figure 1), and by using the following formula: Area $=\sum \mathrm{P} \times \mathrm{a} / \mathrm{p}$; where $\sum \mathrm{P}$ was the total points laid on the wound area and a/p, the area surrounded by every four crosses, was considered as the area per point $\left(\mathrm{mm}^{2}\right)[16,17]$. Thereafter, the wound closure rate was calculated as: Wound closure rate $(\%)=$ (area at visit $1-$ area at each visit $) /$ area at visit 1) $\times 100$.

In a systematic random sampling manner, nine pieces of the skin samples, each about $1 \mathrm{~mm}^{2}$, were cut and prepared for stereological analysis. The pieces were embedded in a cylindrical paraffin block. Isotropic uniformly random (IUR) sections of the blocks with $5 \mu \mathrm{m}$ and $15 \mu \mathrm{m}$ thickness were obtained (Figure 2) and stained with both Hedenhain's azan and hematoxylin-eosin stains. Microscopic analyses of the dermis were done using a video-microscopy system made up of a microscope (E-200; Nikon ${ }^{\text {ix }}$; Japan) linked to a video camera and a flat monitor. The volume densities of the collagen bundles, vessels, and hair follicles $(\mathrm{Vv}$; fraction of the unit volume of the dermis which is occupied by the collagen bundles, vessels, or hair follicles) were estimated by using the point counting method and the following formula: $\mathrm{Vv}_{\text {(collagen }}$ or vessel or hair follicle/dermis) $=\mathrm{P}_{\text {(collagen or vessel or hair follicle) }} / \mathrm{P}_{\text {(dermis) }}$; where $\mathrm{P}_{\text {(collagen or vessel }}$ or hair follicle) was the number of points hitting the profiles of the collagen

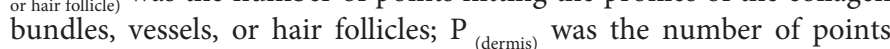
hitting the reference visible field (dermis) (Figure 3).

The length density (Lv) and the mean diameters of the vessels were estimated at final magnification of $450 \times$ by employing the $5 \mu \mathrm{m}$ thickness slides and the following formula: $\mathrm{L}_{\mathrm{v}}=2 \times \sum \mathrm{Q} /\left((\mathrm{a} / \mathrm{f}) \times \sum \mathrm{f}\right)$; where " $\Sigma Q$ " was the total number of the vessel profiles counted in each rat's sample, $(\mathrm{a} / \mathrm{f})$ was the area of the counting frame, and " $\Sigma \mathrm{f}$ " was the total number of the frames counted per animal (Figure 4).

The numerical density (Nv; number of the cells per unit volume of the dermis) of the fibroblasts was estimated by employing the $15 \mu \mathrm{m}$ slides, the "optical dissector" method [16], and the following formula: $\mathrm{Nv}=\Sigma \mathrm{Q} / \Sigma \mathrm{A} \times \mathrm{h}$; where " $\Sigma \mathrm{Q}$ " was the number of the nuclei coming into focus in the dissector height, " $\sum \mathrm{A}$ " was the total area of the counting frame in all microscopic fields, and "h" was the height of dissector within which the nuclei were counted. The upper and the lower $5 \mu \mathrm{m}$ were considered as "area of safety" (Figure 5).

Statistical analysis of the data: The data were collected, analyzed, and reported as mean and standard deviation (mean $\pm \mathrm{SD}$ ). Besides, the statistical comparisons between the groups were carried out by the SPSS statistical software (v.16.0). One-way analysis of variance

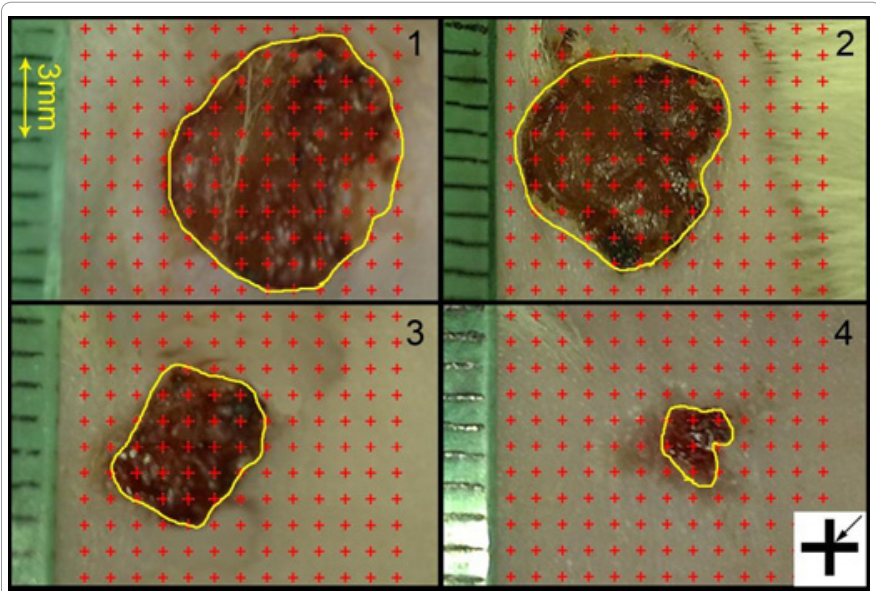

Figure 1: Digital photographs were captured from the wound surfaces every other day to measure the wound area. The total number of points within the wound borders (yellow line) was counted. At the corner of this figure, a cross is presented. The right upper corner of the cross is considered as the point (arrow), and it is counted only if the right upper corner hits the wound surface.

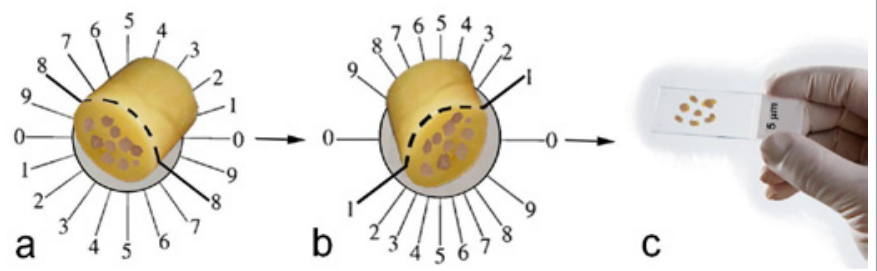

Figure 2: The orientator method to generate IUR sections. (Left): Each cylindrical block was placed on a circle, which was divided into 10 equal distances, a random number between zero and nine was selected and the block was sectioned with a blade in that direction (here 8). (Centre): The sectioned surface of the block was placed on the 0-0 direction again and the next cut was done by selecting another random number (here 1). 
Citation: Ashkani-Esfahani S, Emami Y, Esmaeilzadeh E, Bagheri F, Namazi MR (2012) Glucosamine Enhances Tissue Regeneration in the Process of Wound Healing in Rats as Animal Model: A Stereological Study. J Cytol Histol 3:150. doi:10.4172/2157-7099.1000150

Page 3 of 5

(ANOVA), Tukey's post test, and Mann-Whitney U-test were used in order to analyze the data. Moreover, $\mathrm{P} \leq 0.05$ was considered as statistically significant.

\section{Results}

\section{Area of the wounds}

The mean initial area of the wounds was $104.63 \pm 9.34 \mathrm{~mm}^{2}$ and there were no significant differences among the three groups regarding the primary wound areas. Nevertheless, the rate of wound closure in $2 \%$ GA treated group was significantly higher in comparison to the control and the gel base treated groups $(\mathrm{P}<0.05)$ (Figure 6). Of course, the rate of wound closure in the group treated with the gel base was similar to that of the control group $(\mathrm{P}>0.1)$.

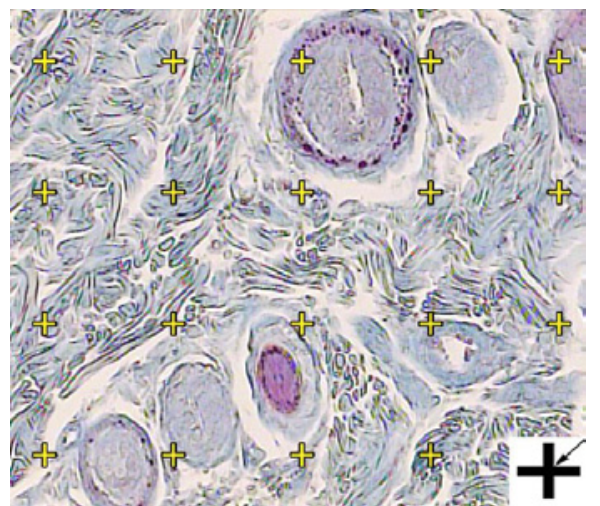

Figure 3: The volume densities $(\mathrm{Vv})$ of the collagen bundles, vessels and hair follicles were estimated by using a grid of points (designed at Histomorphometry \& Stereology Research Centre, Shiraz University of Medical Sciences) on the live image of dermis. The total number of points hitting the collagen bundles or vessels or hair follicles is counted and divided by the total number of points hitting the reference space (dermis). The right upper corner of the cross, presented at the right corner of this figure, is considered as the point (arrow), and it is counted only if the right upper corner hits the tissue. (Hedenhain's azan stain) $(\times 450)$

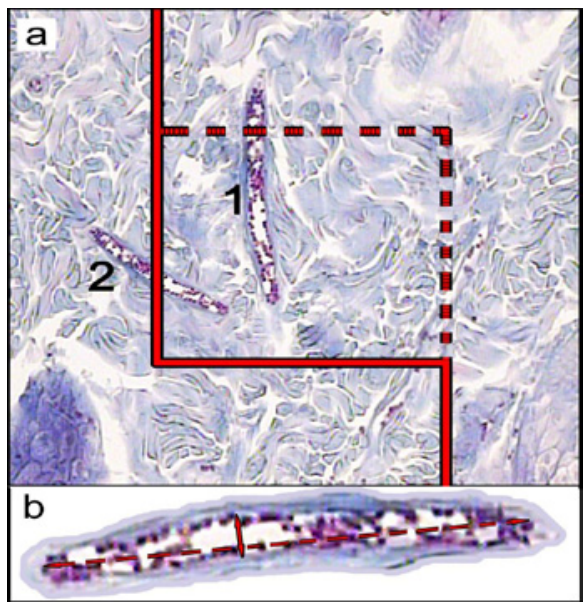

Figure 4: (a) An unbiased counting frame was laid randomly on the monito image of wound dermis at final magnification of 450 for estimation of the length density (LV) and mean diameter of the vessels. Any vessel lied in the counting frame (1), or touched the inclusion borders (dotted lines) were selected. The vessels touched the exclusion borders (bold continuous lines), were ignored (2). (b) The short axis of each vessel (short double arrow) was measured as the mean diameter. (Hedenhain's azan stain; × 450).

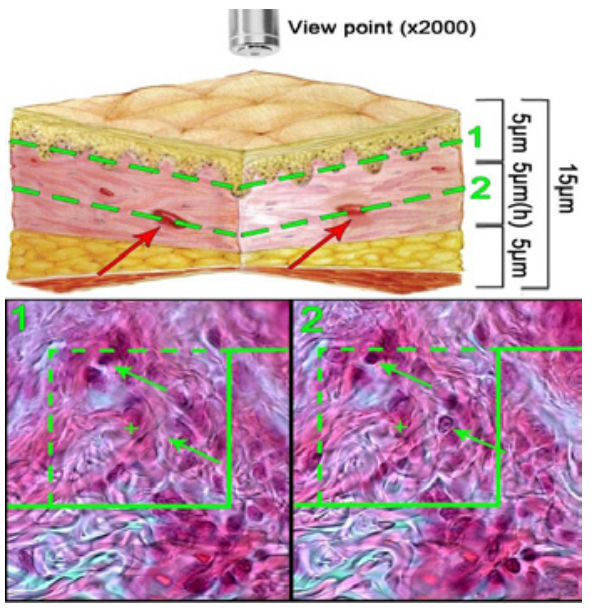

Figure 5: An unbiased counting frame is superimposed on the monitor image of the $15 \mu \mathrm{m}$ sections to estimate the numerical density $(\mathrm{Nv})$ of the fibroblasts. The traveling height was monitored by a microcator. The range of height that the fibroblasts came into maximal focus was considered as height of disector. (1): The nuclei are unclear at the first $5 \mu \mathrm{m}$ optical section. (2): As above, any nucleus lied in the counting frame or touched the inclusion borders (green dotted lines) and did not touch the exclusion borders (bold green lines) and come into maximal focus within the next traveling $5 \mu \mathrm{m}$ optical section (h) are counted (the two arrows). (Hedenhain's azan stain; $\times 2000$ ).

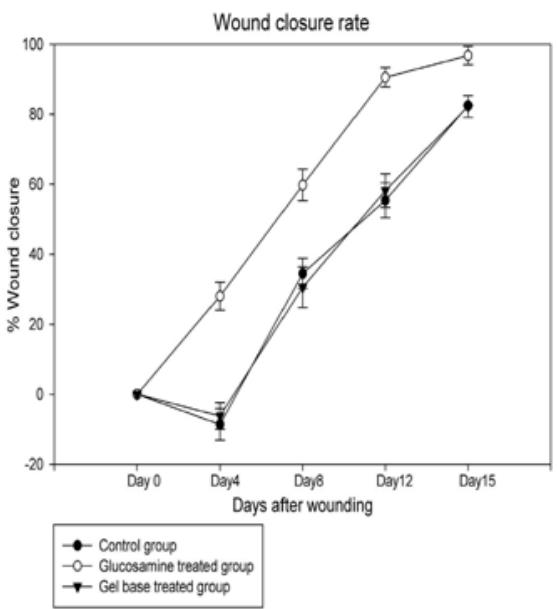

Figure 6: Effect of Glucosamine on wound closure rate in laboratory rats Control, Gel base treated and glucosamine-treated wounded rats. Each point represents the mean $( \pm S E)$ of twelve wounds. Wound closure rate was significantly increased in Glucosamine-treated rats compared with control group and gel base group $(P<0.05)$.

\section{Fibroblast population}

Numerical density of the fibroblasts (Nv) in the dermis of the GA treated group was significantly higher than that of the control and the base groups. Numerical density of the fibroblasts in GA -treated groups was reported as $45.3 \%$ higher than the controls $(\mathrm{P}=0.003)$ and $45.6 \%$ higher than the base group. $(\mathrm{P}=0.003)$ (Table 1, Figure 7).

\section{Volume density of the collagen bundles and hair follicles}

The volume density of the collagen bundles was $32.3 \%$ and $46.5 \%$ higher in the GA-treated group compared to the control and the base groups, respectively, which both were statistically significant $(\mathrm{P}<0.001)$ (Table 1) (Figure 7). Consequently, the volume density of hair follicles 
was higher in the GA-treated group in comparison to the control (66.1\%; $\mathrm{P}=0.043)$ and the base $(210 \% ; \mathrm{P}=0.001)$ group (Table 1) (Figure 7).

\section{Volume density, length density and diameter of the vessels}

The volume density of the vessels in the GA group was $11.7 \%$ $(\mathrm{P}=0.985)$ and $58.3(\mathrm{P}=0.818)$ higher than the control and the base group, respectively. In addition, the length density of the vessels was lower in the GA treated group compared to the control $(\mathrm{P}=0.873)$ and the base group $(\mathrm{P}=0.875)$. However, no significant differences were observed regarding the means of the vessel diameters $(\mathrm{P}=0.99)$ (Table $1)$.
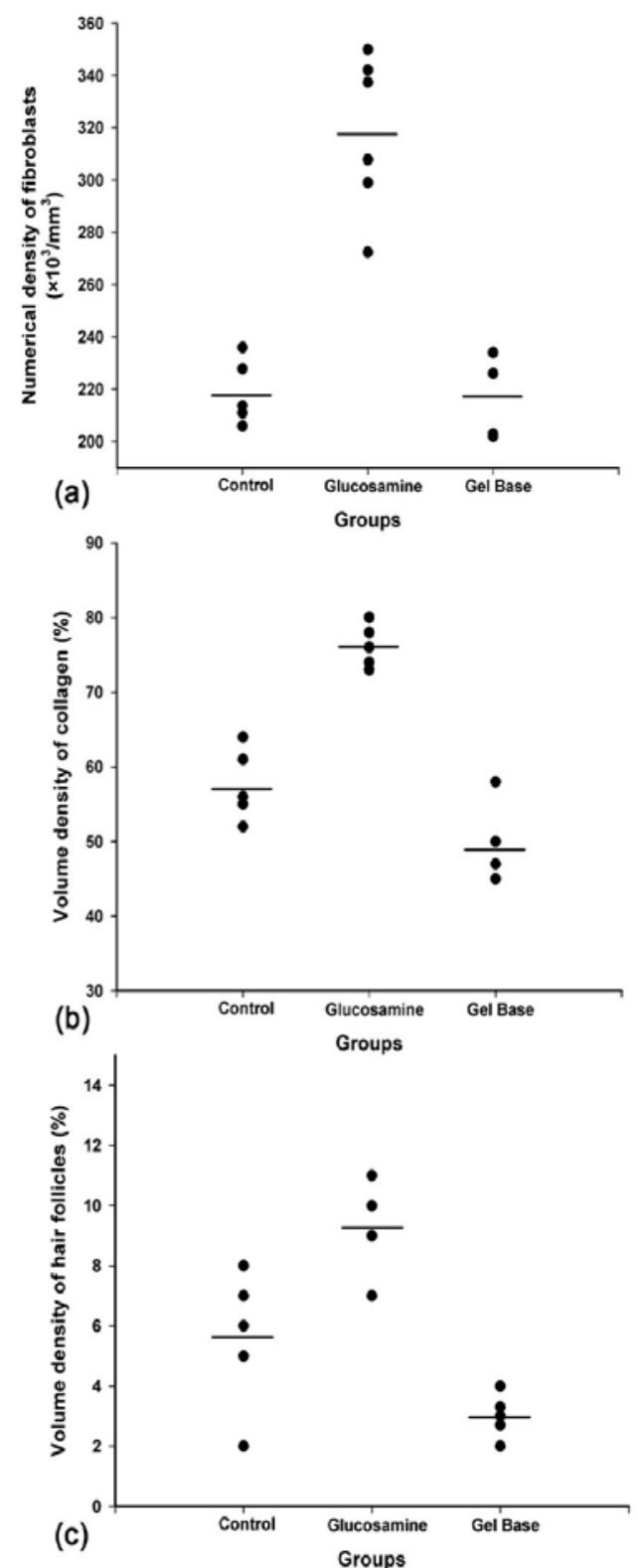

Figure 7: Effect of Glucosamine on wound closure rate in laboratory rats; Control, Gel base treated and glucosamine-treated wounded rats. Plots: (a) numerical density of the fibroblasts $\left(\times 10^{3} / \mathrm{mm}^{3}\right)$, (b) volume density of the collagen bundles (\%), and (c) volume density of the hair follicles (\%) in the dermis.

\section{Discussion}

Considering the importance of treating the skin wounds, finding more beneficial agents to enhance and improve the wound healing process have always been a concern for the researchers. GA is an aminomonosaccharide, made from chitin or chitosan, that is present in all human tissues and is produced by the addition of an amino group to glucose [12]. Many studies have been conducted on the wound healing effects of chitin and chitosan Topical administration of chitosan was reported to accelerate the infiltration of inflammatory cells such as polymorphonuclear leukocytes (PMN) and macrophages [18]. Chitosan was also exhibited to stimulate the secretion of inflammatory mediators which are involved in the process of wound healing such as tumor necrosis factor alpha (TNF- $\alpha$ ), Interleukin(IL)-1, IL-2, IL-8, IL-12, macrophage inflammatory protein (MIP)-1 $\alpha$, MIP-1 $\beta$, transforming growth factor (TGF)- $\beta 1$, and platelet derived growth factor (PDGF) $[19,20]$. Previous studies also reported that topical chitosan consumption improved the granulation tissue formation in skin wounds by increasing the production of collagen bundle synthesis (collagen type III) [18]. Synthesis of Osteopontin, a glycosylated phosphoprotein which is a ligand for the $\alpha v \beta 3$ integrin, was stimulated by chitosan in migratory PMNs and was seen to have a significant role in the evolution of wound healing [18]. Complement activation and chemoattractant abilities of chitosan were the other mechanism of enhancing the PMN activity by this agent [21,22]. Moreover, chitosan promoted the angiogenesis indirectly through complement activation which led to secretion of IL-8, a cytokine-induced neutrophil chemoattractant, by fibroblasts in vivo. Chitin and chitosan and their derivatives indirectly enhanced the fibroblast proliferation through stimulating the inflammatory cells to secrete IL-1 and some other mediators in vitro $[6,23]$ and in vivo [18]; however, an in vitro study by Mori et al. [24] demonstrated that chitin and its derivatives such as GA $(500 \mu \mathrm{g} / \mathrm{ml})$ not only have no stimulatory effect on fibroblast proliferation but also reduced the rate of fibroblast proliferation in high doses, thus, we selected lower dosages of GA in order to prevent the possible negative effects of this substance on fibroblast proliferation, as a necessary requirement in the healing process, and to evaluate the healing effect in lower doses. In a clinical trial, Azad and his colleagues showed that efficient adherence, blood supplying, granulation tissue formation, and re-reepithelialization of the wound could be promoted by using chitosan mesh membrane as a wound dressing [25].

GA, as a derivative of chitin and chitosan, was shown to have an immunomodulatory influence in atopic dermatitis like skin lesion in vitro by reducing T-helper 2 cells released cytokines such as IL- 4 and IL-5 that improved the healing process of these lesions [26]. Hwang et al. [27] showed that GA increased the viability and inhibited reactive oxygen species production in immortalized human skin fibroblasts (HS68) cells exposed to UVB irradiation; pre-treatment of HS68 cells with GA inhibited UVB-induced production of the MMP-1 and MMP13 collagenases. This antioxidative activity of GA, which is beneficial for wound healing, was also shown by some other investigations $[10,28]$. Moreover, many studies suggested GA as an anti-inflammatory agent $[29,30]$; focusing on skin, Hong and colleagues [29] revealed that GA has anti-inflammatory ability through down-regulating COX-2 and MMP13 expression by human skin fibroblasts. Furthermore, granulation tissue formation and angiogenesis in the wound site were seen to be promoted by the topical administration of poly- $\mathrm{N}$-acetyl-glucosamine 
[31]. Takemoto et al. [32] declared that GA dose-dependently enhances the synthesis of osteopontin in rat's aortic smooth muscle which is not still examined in the skin. Although there were many studies conducted on different aspects of dermatological effects of GA, chitin, and chitosan [33], Poly-N-acetyl glucosamine [31], N-acetyl glucosamine [14], a few investigations were found on GA and its wound healing effects via topical administration [7]. Results of this study demonstrated that GA significantly intensified collagen bundle synthesis and hair follicle production. Unlike the previous reports on inhibitory impacts of high dose GA on fibroblast proliferation, the population of fibroblasts was improved with the $2 \%$ GA gel consumption. Although GA treated group had increased the volume density of vessels and also the mean diameter, the overall impact on vascularization was not significant. Moreover, the wound closure rate, which is of the main criteria of skin repair, was significantly augmented by topical GA administration.

Overall, GA and chitosan have many effects and targets in common suggesting that GA is the major component of chitosan and chitin related to the wound healing effects of these agents. Briefly, according to the previous reports on different aspects of GA effects and the results of this experimental study, it was demonstrated that GA has the potent agent in the process of wound healing that can be consumed even as a treatment; however, still further evaluations on its mechanisms of action, feasible adverse effects and possibility of being administered clinically should be pointed by future investigations.

\section{Acknowledgments}

This study was supported by Shiraz University of medical sciences, Shiraz, Iran. Authors wanted to appreciate Dr. M. Vahedi, Mr. Sarayi and all the personne of Stem Cell and Transgenic Technology Research Center, Ms. Keivanshokooh and Research Improvement Center of Shiraz University of Medical Sciences, Shiraz, Iran.

\section{References}

1. Martin $P$ (1997) Wound healing-aiming for perfect skin regeneration. Science 276: 75-81.

2. Ono I, Gunji H, Zhang JZ, Maruyama K, Kaneko F (1995) A Study of cytokine in burn blister fluid related to wound healing. Burns 21: 352-355

3. Cochrane C, Rippon MG, Rogers A, Walmsley R, Knottenbelt D et al. (1999) Application of an in vitro model to evaluate bioadhesion of fibroblasts and epithelial cells to two different dressings. Biomaterials 20: 1237-1244.

4. Clark RA (1993) Regulation of fibroplasia in cutaneous wound repair. Am J Med Sci 306: 42-48.

5. Anderson JW, Nicolosi RJ, Borzelleca JF (2005) Glucosamine effects in humans: a review of effects on glucose metabolism, side effects, safety considerations and efficacy. Food Chem Toxicol 43: 187-201.

6. Sal'nikova SI, Drogovoz SM, Zupanets IA (1990) [The liver-protective properties of D-glucosamine]. Farmakol Toksikol 53: 33-35.

7. McCarty MF (1996) Glucosamine for wound healing. Med Hypotheses 47: 273 275.

8. Lippiello L (2003) Glucosamine and chondroitin sulfate: biological response modifiers of chondrocytes under simulated conditions of joint stress. Osteoarthritis Cartilage 11: 335-342.

9. Towheed T, Maxwell L, Anastassiades TP, Shea B, Houpt J et al. (2005) Glucosamine therapy for treating osteoarthritis (Review). Cochrane Database Syst Rev 18: CD002946.

10. Xing R, Liu S, Guo Z, Yu H, Li C, et al. (2006) The antioxidant activity of glucosamine hydrochloride in vitro. Bioorg Med Chem 14: 1706-1709.

11. Yan Y, Wanshun L, Baoqin H, Changhong W, Chenwei F, et al. (2007) The antioxidative and immunostimulating properties of D-glucosamine. In Immunopharmacol 7: 29-35.
12. Bissett DL (2006) Glucosamine: an ingredient with skin and other benefits. J Cosmet Dermatol 5: 309-315.

13. Weindl G, Schaller M, Schafer-Korting M, Korting HC (2004) Hyaluronic acid in the treatment and prevention of skin diseases: molecular, biological, pharmaceutical and clinical aspects. Skin Pharmacol Physiol 17: 207-213.

14. Sayo T, Sakai S, Inoue S (2004) Synergistic effect of N-acetylglucosamine and retinoids on hyaluronan production in human keratinocytes. Skin Pharmaco Physiol 17: 77-83.

15. Kikuchi K, Matahira Y (2002) Oral N-acetylglucosamine supplementation improves skin conditions of female volunteers: clinical evaluation by a microscopic three-dimensional skin surface analyzer. J Appl Cosmetol 20: 143152.

16. Gundersen HJ, Bendtsen TF, Korbo L, Marcussen N, Møller A, et al. (1988) Some new, simple and efficient stereological methods and their use in pathological research and diagnosis. APMIS 96: 379-394

17. Ashkani-Esfahani S, Imanieh $\mathrm{MH}$, Khoshneviszadeh $\mathrm{M}$, Meshksar A Noorafshan A et al. (2012) The healing effect of arnebia euchroma in second degree burn wounds in rat as an animal model. Iran Red Crescent Med J 14 70-74.

18. Ueno H, Yamada H, Tanaka I, Kaba N, Matsuura M, et al. (1999) Accelerating effects of chitosan for healing at early phase of experimental open wound in dogs. Biomaterials 20: 1407-1414.

19. Ueno H, Mori T, Fujinaga $\mathrm{T}$ (2001) Topical formulations and wound healing applications of chitosan. Adv Drug Deliv Rev 52: 105-115.

20. Cassatella MA (1995) The production of cytokines by polymorphonuclear neutrophils. Immunol Today 16: 21-26.

21. Suzuki Y, Okamoto Y, Morimoto M, Sashiwa H, Saimoto H, et al. (2000) Influence of physico-chemical properties of chitin and chitosan on complement activation. Carbohyd Polym 42: 307-310.

22. Usami Y, Okamoto Y, Minami S, Matsuhashi A, Kumazawa NH, et al. (1994) Migration of canine neutrophils to chitin and chitosan. J Vet Med Sci 56: 12151216.

23. Nishimura K, Nishimura S, Nishi N, Saiki I, Tokura S, et al. (1984) Immunological activity of chitin and its derivatives. Vaccine 2: 93-99.

24. Mori T, Okumura M, Matsuura M, Ueno K, Tokura S, et al. (1997) Effects of chitin and its derivatives on the proliferation and cytokine production of fibroblasts in vitro. Biomaterials 18: 947-951.

25. Azad AK, Sermsintham N, Chandrkrachang S, Stevens WF (2004) Chitosan membrane as a wound-healing dressing: characterization and clinical application. J Biomed Mater Res B Appl Biomater 69: 216-222.

26. Kim CH, Cheong KA, Park CD, Lee AY (2011) Glucosamine improved atopic dermatitis-like skin lesions in NC/Nga mice by inhibition of Th2 cell development. Scand J Immunol 73: 536-545.

27. Hwang YP, Kim HG, Han EH, Choi JH, Park BH, et al. (2011) N-Acetylglucosamine suppress collagenases activation in ultraviolet B-irradiated human dermal fibroblasts: Involvement of calcium ions and mitogen-activated protein kinases. J Dermatol Sci 63: 93-103.

28. Xing R, Liu S, Wang L, Cai S, Yu H, et al. (2009) The preparation and antioxidant activity of glucosamine sulfate. Chin J Oceanol Limnol 27: 283-287.

29. Hong H, Park YK, Choi MS, Ryu NH, Song DK, et al. (2009) Differential downregulation of COX-2 and MMP-13 in human skin fibroblasts by glucosaminehydrochloride. J Dermatol Sci 56: 43-50.

30. Nagaoka I, Igarashi M, Hua J, Ju Y, Yomogida S, et al. (2011) Recent aspects of the anti-inflammatory actions of glucosamine. Carbohyd Polym 84: 825-830.

31. Yang HJ, Yoon CS (2008) Effects of Poly-N-acetyl Glucosamine(pGlcNAc) Patch on Wound Healing in $\mathrm{db} / \mathrm{db}$ Mouse. J Korean Soc Plast Reconstr Surg 35: $121-126$

32. Takemoto M, Yokote K, Yamazaki M, Ridall L, Butler WT, et al. (1999) Enhanced expression of osteopontin by high glucose in cultured rat aortic smooth muscle cells. Biochem Biophys Res Commun 258: 722-726

33. Azad AK, Sermsintham N, Chandrkrachang S, Stevens WF (2004) Chitosan Membrane as a Wound-Healing Dressing:Characterization and Clinica Application. J Biomed Mater Res B Appl Biomater 69B: 216-222. 